\title{
Advanced Feature Extraction and Dimensionality Reduction for Unmanned Underwater Vehicle Fault Diagnosis
}

\author{
Abed, W
}

http://hdl.handle.net/10026.1/8017

\subsection{9/CONTROL.2016.7737596}

The 2016 UKACC International Conference on Control (UKACC Control 2016)

IEEE

All content in PEARL is protected by copyright law. Author manuscripts are made available in accordance with publisher policies. Please cite only the published version using the details provided on the item record or document. In the absence of an open licence (e.g. Creative Commons), permissions for further reuse of content should be sought from the publisher or author. 
This is the accepted version (i.e. the last author-generated version)

$$
\text { of a paper published as }
$$

Wathiq Abed, Riccardo Polvara, Yogang Singh, Sanjay Sharma, Robert Sutton, Daniel Hatton, Andrew Manning and Jian Wan. "Advanced feature extraction and dimensionality reduction for unmanned underwater vehicle fault diagnosis". In The 2016 UKACC International Conference on Control (UKACC Control 2016), IEEE, Danvers, 2016.

doi:10.1109/CONTROL.2016.7737596

\section{Copyright (C) 2016 IEEE}

For subscribers, the final, published version can be found through IEEE Xplore here. 


\title{
Advanced Feature Extraction and Dimensionality Reduction for Unmanned Underwater Vehicle Fault Diagnosis
}

\author{
Wathiq Abed, Riccardo Polvara, Yogang Singh, Sanjay Sharma, Robert Sutton, Daniel Hatton, Andrew \\ Manning and Jian Wan \\ Autonomous Marine Systems Research Group, School of Marine Science and Engineering \\ University of Plymouth \\ Plymouth, UK
}

\begin{abstract}
This paper presents a novel approach to the diagnosis of blade faults in an electric thruster motor of unmanned underwater vehicles (UUVs) under stationary operating conditions. The diagnostic approach is based on the use of discrete wavelet transforms (DWT) as a feature extraction tool and a dynamic neural network (DNN) for fault classification. The DNN classifies between healthy and faulty conditions of the trolling motor by analyzing the stator current and vibration signals. To overcome feature redundancy, which affects diagnosis reliability, the Orthogonal Fuzzy Neighbourhood Discriminant Analysis (OFNDA) approach is found to be the most effective. Four faulty conditions were analyzed under laboratory conditions, and the results obtained from experiment demonstrate the effectiveness and reliability of the proposed methodology in classifying the different faults faster and more accurately.
\end{abstract}

Keywords - dimensionality reduction; dynamic neural network; fault diagnosis; feature extraction

\section{INTRODUCTION}

Unmanned underwater vehicles (UUVs) are now being employed by the scientific, offshore and naval sectors to perform a multitude of different tasks [1]. As a consequence of their success, these sectors are now demanding longer mission lengths coupled with increasing vehicle autonomy. With an escalation in autonomy comes the need for higher reliability in such vehicles, in order for them to better cope with unexpected events. Hence there is a growing interest in the use of fault detection and diagnostic techniques in UUVs.

This paper presents a novel approach to the diagnosis of unbalanced load faults in an electric thruster motor. Such motors are typically employed in propulsion systems for small electric powered craft such as UUVs owing to their high efficiency, size and weight. For the study herein, the propulsion system considered consists of two propellers powered by a set of 24V 334N Minn Kota Riptide transom mounted saltwater trolling motors. This motor is conventionally used in surface vehicles but can be extended to be used in UUVs.

After data collection of the essential sensor signals, features are often extracted and selected to analyze the signals from all these embedded sensors to assess the condition of the system by the indirect method. Feature extraction is usually the first step in any pattern recognition system [2].

Dynamic properties of machine are dependent on current and vibration signals. To overcome the limitations of time domain and frequency domain feature extraction techniques, time-frequency domain is suitable to indicate transient events in the signal [2]. Discrete wavelet transform (DWT) has been proposed as one of the powerful methods for this task [12]. DWT has the ability to explore signal features with partial characteristics and analyze signals with different time and frequency resolutions. Various approaches have been developed to optimize mother wavelet selection. Jha [3] compared between several mother wavelet functions and found a Daubechie optimal mother wavelet for rolling element bearing and gear fault diagnosis, while Rafee [4] found the selected mother wavelet for a specific signal might be not suitable for other signals. In this paper the standard deviation (STD) and minimum description length (MDL) criteria is applied to select the optimum level of a mother wavelet.

Irrelevant features will affect the learning process, by increasing the computational cost and sample size, and may furthermore lead to over-fitting. In order to increase the robustness of the classifier and to reduce the data processing load, dimensionality reduction is necessary. As such, the main goal of feature subset reduction is to reduce the number of features used in classification, without compromising the accuracy.

The orthogonal fuzzy neighborhood discriminative analysis approach (OFNDA) is proposed as feature reduction techniques: basically, OFNDA works to maximize the distance between features belonging to different classes while minimizing the distance between features in the same class $[5]$.

Moving on from dimensionality reduction to feature classification AI techniques such as feed forward neural network (NN), support vector machine (SVM) and adaptive neural fuzzy inference system (ANFIS) have been used for classifying faults in motors [13]. Among the various pattern recognition approaches to condition monitoring and fault 
diagnosis of electrical machinery, NNs have been commonly used to classify training patterns from samples [6].

Dynamic Neural Networks (DNN) are more versatile than their static counterparts, and provide the capability to learn the dynamics of complicated nonlinear systems that conventional static neural networks cannot model [7]. DNNs have been successfully applied in fault diagnosis [6] and studies have shown that their use can improve the fault prediction accuracy of electric motor condition monitoring systems.

\section{EXPERIMENTAL SETUP}

Hard surfaces can damage blades partly or fully and can imbalance the operation of a trolling motor, causing significant damage to the internal parts.

The proposed technique was tested on an experimental set up to show the behaviour of the trolling motor under normal operating condition and four faulty conditions in $10 \%$ blade cut (F1), $25 \%$ blade cut (F2), half (F3) and full cut (F4), as shown in Fig. 1. Matlab was used to change the duty cycle of the PWM signals and the motor was powered from a $24 \mathrm{~V}$ battery supply.

Data is gathered at a sampling rate of $3 \mathrm{kHz}$ for duration of $30 \mathrm{sec} ; 90000$ sample points were obtained for current and vibration at variable rotor speeds. Fig. 2 shows schematic diagram of the laboratory set-up for the trolling motor fault diagnosis. A linear current sensor is used to measure the stator current and an accelerometer is mounted on the flat surface of the propeller to record the vibration. Sensor outputs were logged to a PC via a data acquisition card (NI USB-6009 multifunction).

\section{FEATURES EXTRACTION FOR FAULt DiAgNOSIS}

To extract the useful information, DWT, a signal analysis method that provides the time and frequency information of the signal was applied. The information that cannot be readily seen in the time domain can be observed in the frequency domain. It has the ability to explore signal features with partial characteristic and analyze signals with different time and frequency resolutions.

The DWT approach is successfully applied to detect and locate faults, together with the identification of the severity of the faults. The same approach can be extended to identify the other faults. The signal is passed through a series of high pass filters $\left(H_{n}\right)$ to analyse the high frequencies, and it is also passed through a series of low pass filters $\left(L_{n}\right)$ to analyse the low frequency components of the signal [8], as shown in Fig.3. At each decomposition level, the half band filters produce signals spanning only half the frequency band. This doubles the frequency resolution as the uncertainty in frequency is reduced by half with this approach; the time resolution becomes arbitrarily good at high frequencies, while the frequency resolution becomes arbitrarily good at low frequencies.

The correlation between the signal and the wavelet at each level of scaling and shifting is termed the wavelet coefficient. The resolution of the signal, which is a measure of the amount of detail of information in the signal, is changed by the filtering operations, and the scale is changed by altering the size window of signals. Resulting from the DWT decomposition, a set of wavelet energy signals $\left(d_{n}\right)$ and $\left(a_{n}\right)$ are obtained [9].
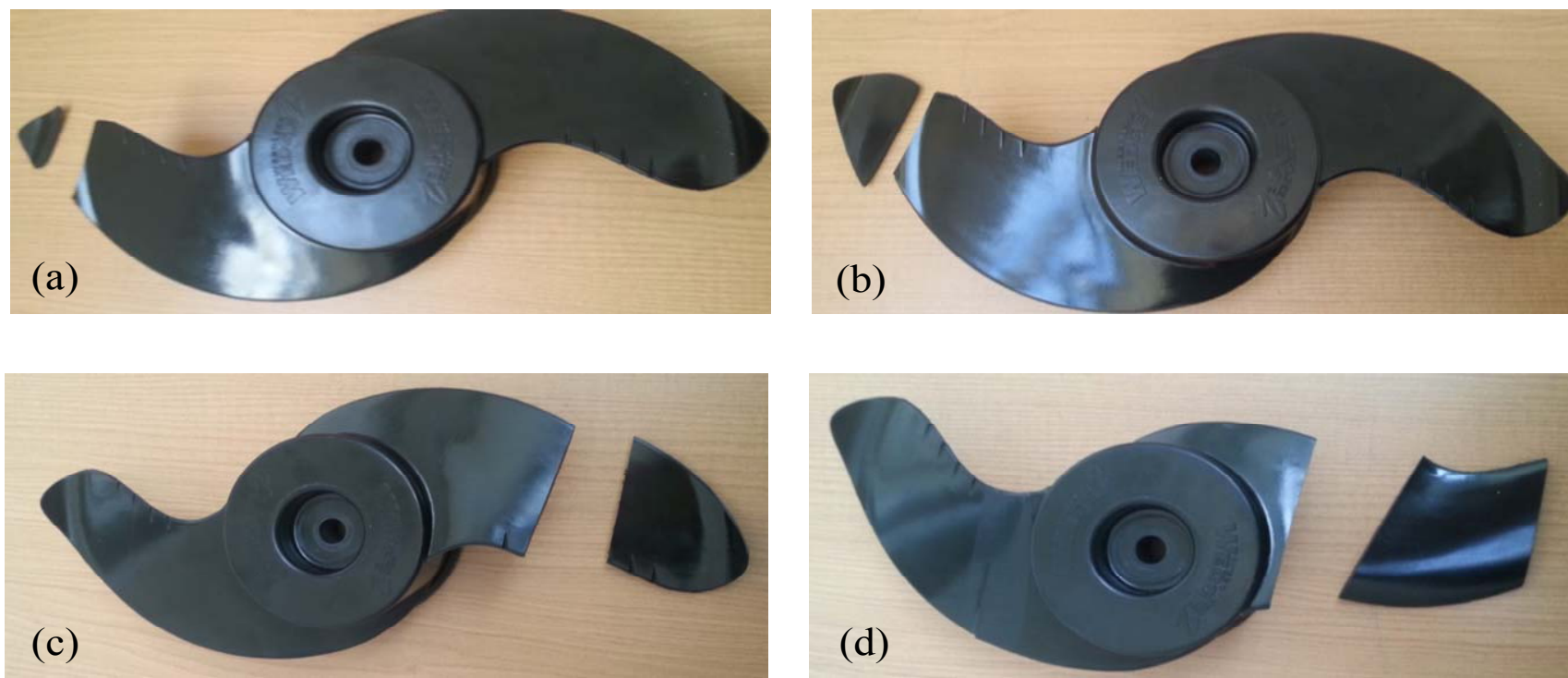

Fig.1. Faults in the blades of the trolling motor (a) $10 \%$ blade cut, (b) $25 \%$ blade cut, (c) half and (d) full cut 


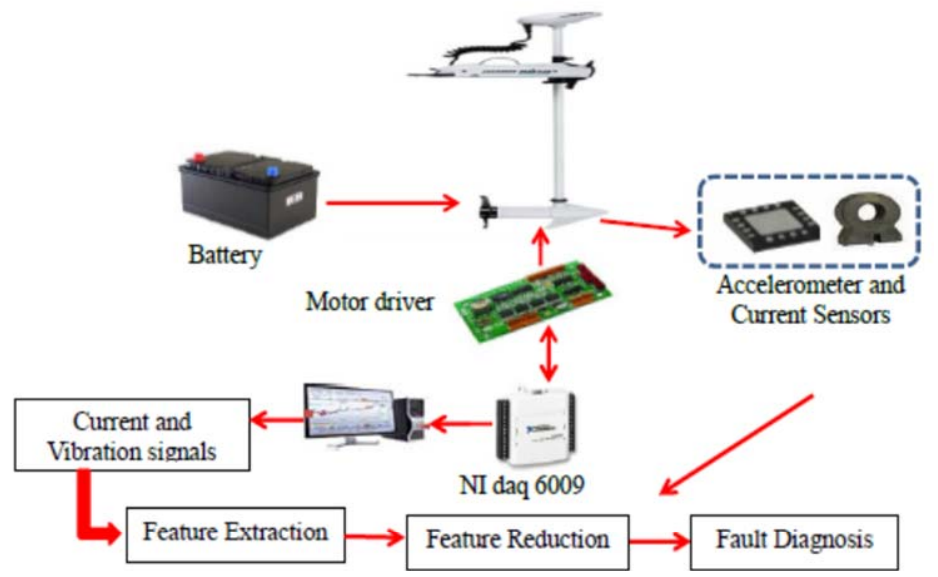

Fig.2. Schematic diagram of the experimental setup

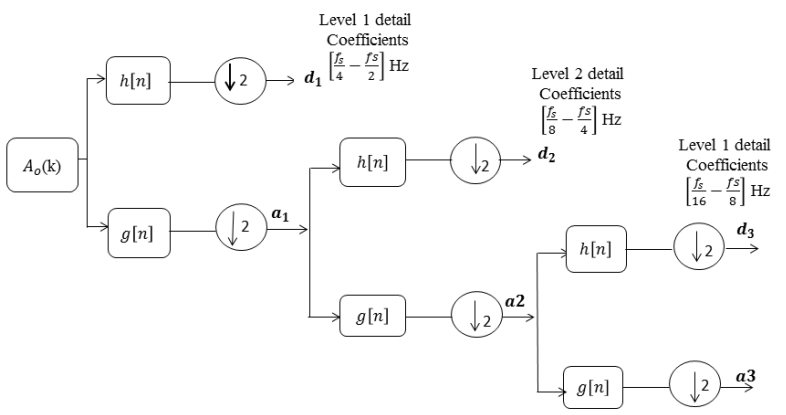

Fig.3. Schematic diagram of discrete wavelet transforms

Before the application of the discrete wavelet transform (DWT), first we have to select the type of mother wavelet and the number of decomposition levels. The choice of wavelet is dictated by the signal characteristics and the nature of the application. Wavelet families vary in terms of several important properties such as speed of convergence to zero when time or frequency goes to infinity, symmetry, regularity etc. Therefore, the criteria for selecting a proper mother wavelet is to have a wavelet function with a sufficient number of non-vanishing moments, in order to represent the salient features of the disturbance [10].

For optimal wavelet analysis selection, STD and MDL data criteria are used. This method is based on the optimal number of wavelet coefficients to be retained for the signal reconstruction. The MDL function of index $m$ (number of coefficients to be retained) and $n$ (number of wavelet filters) is described as [11].

$\operatorname{MDL}(m, n)=\left\{\frac{3}{4} m \log N+\frac{N}{2} \log \left\|\hat{a}_{n}-\hat{a}_{n}^{m}\right\|^{2}\right\}$

where

$$
0<m<N, 1<n<N
$$

where
$\hat{a}_{n}=W n f$ defined as the vector of the decomposition coefficients of the signal $f$ via the wavelet filter $n, \hat{a}_{n}^{m}=\theta^{(m)}$ , $\hat{a}_{n}=\theta^{(m)}(W n f)$ represents the vector that contains $m$ number of coefficients to be retained, and $\hat{a}_{n}$ is a hardthreshold operation, that keeps the $m^{\text {th }}$ largest elements of $\hat{a}_{n}$ in absolute value.

In this paper, the MDL criterion in (1) is tested on the collected data for the available orthogonal or non-orthogonal wavelets in order to select the optimal mother wavelet. As a result, orthogonal wavelet filter 'db12' of the Daubechies family is chosen as the optimal mother wavelet, as illustrated in Table I [10].

The minimum number of decomposition levels necessary for obtaining an approximation signal (a) must be established, so that the upper limit of its associated frequency band is under the fundamental frequency $(f)$ [12]. To determine the optimal wavelet level in this work, dataindependent selection (DIS) approach is considered. The DIS approach is based on the following steps [10]:

$$
2^{-(n L s+1)} f_{s}<f
$$

where, $f_{s}$ is the sampling frequency of the analyzed signal. From this condition, the decomposition level of the approximation signal is the integer $n L s$ :

$$
n L s=\text { integer }\left(\frac{\log \left(\frac{f_{s}}{f}\right)}{\log 2}\right)+2
$$

After calculation according to (3), scale 8 is selected as the decomposition level in this paper. 
TABLE I. MOTHER WAVELET OPTIMIZATION

\begin{tabular}{|c|c|c|c|c|c|}
\hline Filter & MDL & STD & Filter & MDL & STD \\
\hline Haar & 14.78 & 1.13 & sym3 & 15.67 & 1.03 \\
\hline $\mathrm{db} 6$ & 9.88 & 0.82 & sym4 & 11.04 & 0.95 \\
\hline $\mathrm{db} 7$ & 8.17 & 0.79 & coif1 & 9.65 & 1.11 \\
\hline $\mathrm{db} 8$ & 7.43 & 0.75 & coif2 & 13.07 & 0.93 \\
\hline $\mathrm{db} 9$ & 7.7 .23 & 0.71 & coif3 & 10.27 & 0.81 \\
\hline $\mathrm{db} 10$ & 7.13 & 0.70 & sym4 & 11.04 & 0.95 \\
\hline $\mathrm{db} 11$ & 5.13 & 0.70 & bior1. & 14.78 & 1.13 \\
\hline $\mathrm{db} 12$ & 5.04 & 0.67 & bior1. & 7.132 & 1.01 \\
\hline sym2 & 115.63 & 1.11 & bior1. & 11.96 & 0.89 \\
\hline
\end{tabular}

\section{Dimensionality REDUCTION}

Dimensionality reduction is an important task in machine learning, it facilitates classification, compression, and visualization of high-dimensional data by mitigating undesired properties of high-dimensional spaces. After data collection of the essential sensor signals, and feature extraction using DWT, however, a large number of features are usually computed in many industrial applications, and this leads to an increase in time and computational space complexity in the recognition process, in order for the system to make a reasonably accurate deduction.

Khushaba [5] presents OFNDA as a new approach for feature reduction. It works to maximize the distance between features belong to different classes $\left(S_{b}\right)$ whilst minimizing the distance between features in the same class $\left(S_{w}\right)$.OFNDA has been successfully applied to classify 4 classes of rolling element bearing defects and normal conditions, working under variable speed and load conditions.

In the OFNDA process, the first step is to apply principle component analysis, to remove any redundancy that may cause singularity, before starting to discriminate analysis and keeping all principle components, to prevent the loss of any useful information. Then the computation of the proposed fuzzy neighborhood discriminant analysis (FNDA) proceeds by calculating the $S_{w}$ and $S_{b}$ and the transformation matrix is calculated.

$$
\begin{aligned}
& S_{w}=\sum_{i=1}^{c} \sum_{k=1}^{l_{i}} \mu_{i k}\left(X_{k}-X_{i}\right)\left(X_{k}-X_{i}\right)^{T} \\
& S_{b}=\sum_{i=1}^{c} \mu_{i k}\left(U_{i}-X_{X}\right)\left(U_{i}-X_{X}\right)^{T} \\
& G=G_{F N D A} \cdot G_{P C A}
\end{aligned}
$$

where, $\mu_{i k}$ is the membership of pattern $k$ in class $i, X_{k}$ is the $K_{t h}$ sample, and $U_{i}$ is the mean of the patterns that belong to class $i$ and $G_{F N D A}$ is the transformation matrix related to PCA and OFNDA respectively. Figure 4 shows the distinctive features obtained using OFNDA for different faults severities.

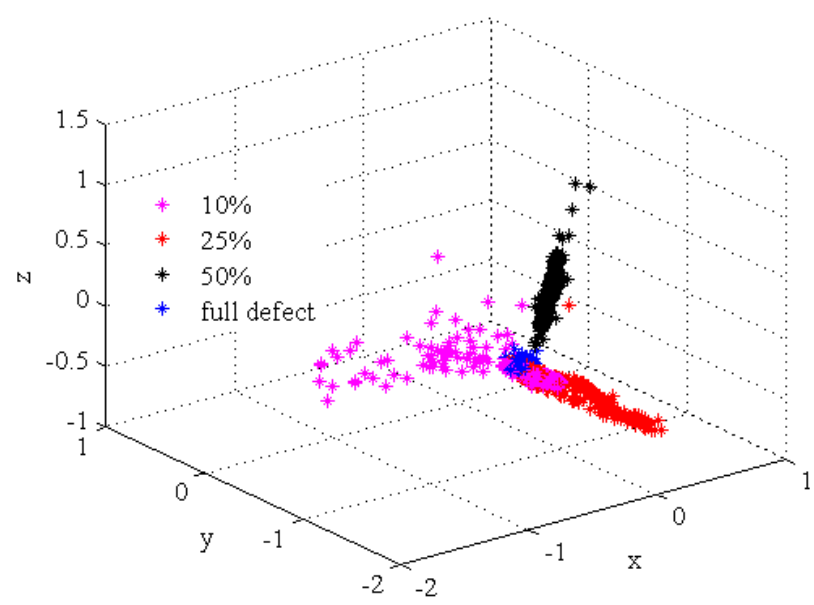

Fig.4. OFNDA features

\section{DNN FOR FAULT CLASSIFICATION}

Most industrial systems are dynamic and nonlinear in nature, and hence during their identification it seems desirable to employ models that can represent their dynamics.

To increase operational reliability and to optimize preventive maintenance, it is necessary to have an efficient real-time tool for analysis and process monitoring. Therefore, in the case of dynamic system identification with this kind of network, an approach based on the feedback from inputs and outputs has to be applied.

DNNs showed themselves more powerful than static NNs, and are useful for electrical machine fault diagnosis [7]. The dynamic neural network that is presented in this paper has a great capability for learning the dynamics of complicated non-linear systems where conventional static neural networks cannot yield and performs acceptable modelling, representation and mapping. In this paper, a Nonlinear Auto-Regressive with exogenous inputs (NARX) model was trained to detect and classify faults. This model is a type of DNN which has a delay line on the input and the output is fed back to the input by another delay line. The DNN has one hidden layer and five outputs. The input consists of the three OFNDA features, $x_{1}^{\text {OFNDA }}, x_{2}^{\text {OFNDA }}$ and $x_{3}^{\text {OFNDA }}$ and the output of the network consists of four units used to indicate a particular bearing fault condition. 


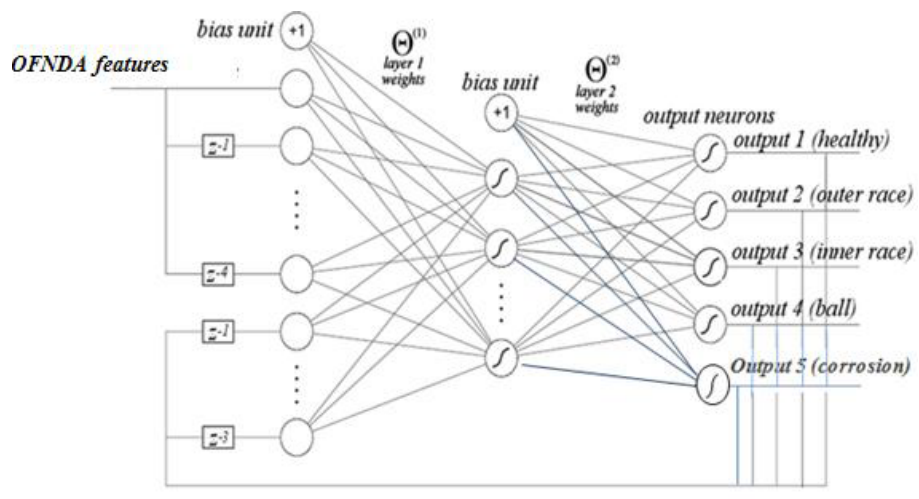

Fig.5 Dynamic neural networks for fault diagnosis process

The input pattern to the network (input layer) at each time $k$ consists of the three input features (present and delayed values), as well as the output feedback, and is formed as:

$$
(k)=\left[\begin{array}{c}
x_{1}^{O F N D A}(k) \\
\vdots \\
x_{1}^{O F N D A}\left(k-n_{d i_{1}}\right) \\
\vdots \\
x_{3}^{O F N D A}(k) \\
\vdots \\
x_{3}^{O F N D A}\left(k-n_{d i_{3}}\right) \\
\widehat{y_{1}}(k-1) \\
\vdots \\
\widehat{y_{1}}\left(k-n_{d o_{1}}\right) \\
\vdots \\
\widehat{y_{4}}(k-1) \\
\widehat{y_{1}}\left(k-n_{d o_{4}}\right)
\end{array}\right]
$$

The network as shown in Figure 5 has tapped delay lines $(d)$ to store previous values of the input, $x(t)$ and output, $y(t)$ sequences. The model can be shown in the following equation:

$$
X(t)=f(x(t-1), \ldots x(t-d), y(t) \ldots y(t-d))
$$

Training of the NN involves trying several different numbers of hidden units to achieve the optimized set for different parameters. MATLAB and Simulink is used to train the NN. The trained NN was evaluated on validation and the test data set. A further test with data obtained under normal and faulty conditions was constructed to simulate the real time operating behaviour of the trolling motor. Supervised learning with Levenberg-Marquardt propagation was used to train the $\mathrm{NN}$ with a training data set.

The process consists of initially assigning some random weights to each of the connections. Forward propagation is then used to obtain the predicted output for each training set sample. All the duration times of the misclassifications are less than $0.7 \mathrm{sec}$. In practice, such misclassification times would not be noticeable and therefore they can be ignored.
Fig.6 shows the overall fault diagnosis test for a motor operating at variable load and speed at $1200 \mathrm{rpm}$. Table II shows that mean classification accuracy using the proposed approach is $97 \%$ which is much better than most of the existing techniques. Many techniques only used one signal as a fault indicator and thus limiting the accuracy in classifying the severity of the faults [15].

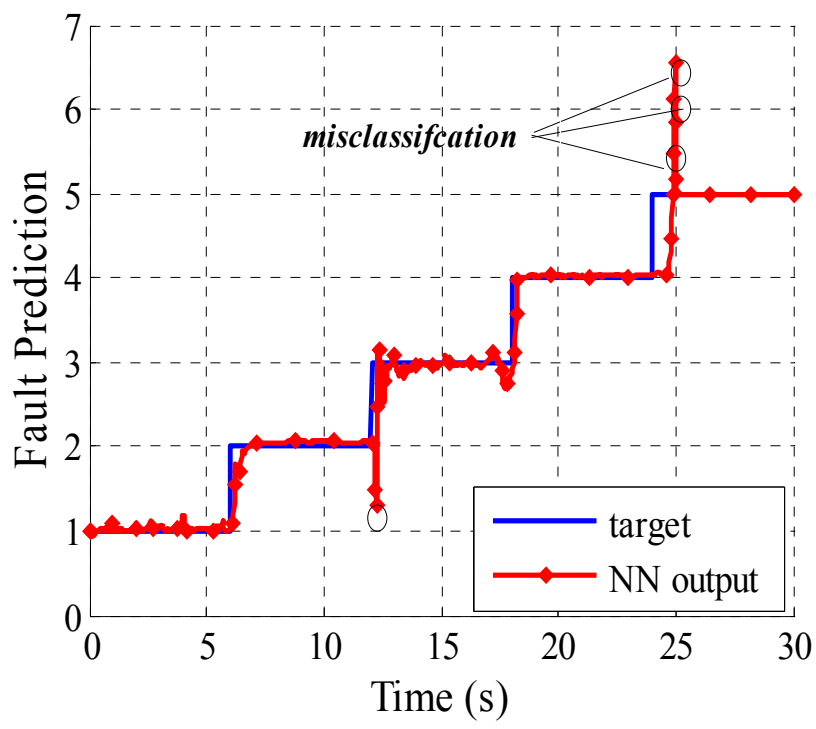

Fig.6 .Overall fault diagnosis test

TABLE II. DNN PERFORMANCE

\begin{tabular}{|c|c|c|}
\hline \multirow{2}{*}{ Speed } & \multicolumn{2}{|c|}{ Classification accuracy } \\
\cline { 2 - 3 } & Validation & Testing \\
\hline Low $(800 \mathrm{rpm})$ & 98.15 & 98.99 \\
\hline High $(1200 \mathrm{rpm})$ & 95.22 & 96.55 \\
\hline
\end{tabular}

\section{CONCLUSIONS}

This paper proposes a new methodology for fault diagnosis of a thruster motor under two unbalanced load operating conditions. A DWT was used as a feature extraction tool to obtain a better resolution of the signal in time and frequency domains and then feature reduction based on OFNDA was applied to obtain the best features for fault classification. These features were then fed into a DNN for classifying the faults and results showed that better classification accuracy was obtained using OFNDA techniques. Further tests simulating the real operating behaviour of the thruster motor under normal and faulty conditions also confirmed the superiority of the proposed method, which can easily be appiled to real time fault detection and classification in UUVs.

\section{REFERENCES}

[1] L. H. Hihara, R. H. Knapp and G. M. Mcmurtry. (2004). Advanced Control of Autonomous Underwater Vehicles . PhD Thesis, University of Hawaii. 
[2] T, Wang, M. Liang, Li, J and W. Chen. (2014). Rolling element ' bearing fault diagnosis via fault characteristic order analysis, Mechanical Systems and Signal Processing,vol. 45,no.1, pp. 139-153.

[3] B. Jha, B.Yadav, M. Rao and H.Yadav. (2013). Selection of Optimal Mother Wavelet for Fault Detection Using Discrete Wavelet Transform, IEEE Transactions on Instrumentation and Measurement, vol. 20 , no. 6 , pp. 2338-2343.

[4] J. Rafiee, W. Tse, A. Harifi and M. Sadeghi. (2009) .A novel technique for selecting mother wavelet function using an intelligent fault diagnosis system, Expert Systems with Applications, vol.36, no. 3, pp. 4862-4875.

[5] N. Khushaba, A. Al-Ani, and A. Al-Jumaily. (2010) .Orthogonal Fuzzy Neighborhood Discriminant Analysis for Multifunction Myoelectric Hand Control, IEEE Transactions on Biomedical Engineering, vol. 57, pp. 1410-1419.

[6] Y. Yang, H. Wang, J. Cheng, and K. Zhang. (2013). A fault diagnosis approach for roller bearing based on VPMCD under variable speed condition, Measurement, vol. 46, pp. 23062312.

[7] W. Xuhong and H. Yigang. (2005). Diagonal recurrent neural network based on-line stator winding turn fault detection for induction motors," In the Eighth International Conference on Electrical Machines and Systems, pp. 2266-2269.

[8] J. Nayak, P. Bhat and R.Acharya (2005). Classification and Analysis of Speech Abnormalities, Elsevier, vol.26, pp. 319327.

[9] J. Antonino, S. Aviyente, E. Strangas and M. Riera (2013). Scale Invariant Feature Extraction Algorithm for the Automatic Diagnosis of Rotor Asymmetries in Induction Motors, IEEE Transactions on Industrial Informatics, vol.9, no.1, pp.100-108.

[10] L. Weilin, A. Monti and F. Ponci (2014). Fault Detection and Classification in Medium Voltage DC Shipboard Power Systems With Wavelets and Artificial Neural Networks, IEEE Transactions on Instrumentation and Measurement, vol. 63, no.11, pp.2651-2665.

[11] Y. Hamid and L. Kawasak (2002). Wavelet-based data compression of power system disturbances using the minimum description length criterion, IEEE Transactions on Power Delivery, vol.17, no.2, pp.460-466.

[12] J. Antonino, M. Riera, J. Roger, F. Martínez and A. Peris. (2006). Application and optimization of the discrete wavelet transform for the detection of broken rotor bars in induction machines, Applied and Computational Harmonic Analysis, vol.21, no.2, pp. 268-279.

[13] W. Li, A. Monti and F. Ponci. (2014). Fault Detection and Classification in Medium Voltage DC Shipboard Power Systems With Wavelets and Artificial Neural Networks, IEEE Transactions on Instrumentation and Measurement,vol. 63, no.11, pp. 2651-2664.

[14] A.Siddique, G.S.Yadava and B.Singh. (2003) .Applications of artificial intelligence techniques for induction machine stator fault diagnostics: review, in Diagnostics for Electric Machines, Power Electronics and Drives, 2003 (SDEMPED 2003) $4^{\text {th }}$ IEEE International Symposium on, vol., no., pp.2934.

[15] W.R.Abed.(2015).Robust Fault Analysis for Permanent Magnet DC Motor in Safety Critical Applications, PhD Thesis, Plymouth University. http://pearl.plymouth.ac.uk/handle/10026.1/3550. 